\title{
Public Health Genomics
}

Abboud, H.E. 431

Abelow, C. 514

Alberg, C. 538

Allen, T. 514

Arar, N. 431

Auclair, J. 424

Bagade, S. 514

Barrett, J.R. 477

Bertram, L. 514

Best, L. 389

Betran, A.P. 514

Boddington, P. 504

Brun, W. 396

Burton, H. 538

Christensen, K.D. 467

Cohn, W.F. 477

Collins, V. 449

Copeland, L.A. 431

Delatycki, M.B. 449

Dolan, S.M. 514

Domchek, S. 440

Dubin, R.A. 514

Duivenvoorden, H.J. 415

Ehrencrona, H. 396

Elward, K.S. 477

Franko, J. 477

Gaudreault, M. 424

Gibson, J. 477

Graubard, B.I. 495

Greene, M.H. 495

Guterbock, T.M. 477

Halbert, C.H. 440

Han, Y. 457

Harrison, M.B. 477

Hollegaard, M.V. 514
Ioannidis, J.P.A. 514

Ioannou, L. 449

Javaher, P. 524

Jayaratne, T.E. 467

Jean, S. 424

Kääriäinen, H. 524

Kardia, S.L.R. 467

Karstanje, J. 415

Kessler, L. 440

Khoury, M.J. 514

Kinzie, M.B. 477

Knaus, W.A. 477

Kristoffersson, U. 524

Kroese, M. 538

Kvale, G. 396

Laberge, L. 424

Lakshman, R. 538

Lee, S. 431

Leman, R.F. 457

Lin, B.K. 514

Liu, Z. 477

Mai, P.L. 495

Massie, J. 449

Mathieu, J. 424

McClaren, B. 449

Menon, R. 514

Merialdi, M. 514

Miesfeldt, S. 477

Møldrup, C. 406

Mullins, I.M. 477

Muna, W. 492

Mutlu, D. 415

Nace, J. 514

Nadeau, M. 389

Newport, M.J. 492

Nippert, I. 524

Noel, P. 431
Nordin, K. 396

Nyoungui, E. 524

Oestergaard, S. 406

Parchman, M. 431

Passchier, J. 415

Pelletier, S.L. 477

Perron, M. 424

Petty, E.M. 467

Prévost, C. 424

Ramesar, R. 492

Richer, L. 424

Roberts, J.S. 467

Ropka, M.E. 477

Rotimi, C.N. 492

Schmidtke, J. 524

Seo, J. 431

Sequeiros, J. 524

Silvey, K. 457

Soller, M. 396

Stopfer, J.E. 440

Tibben, A. 415

Troxel, A.B. 440

Tucker, A.L. 477

Valdez, R. 457

van Elderen, T. 415

Veillette, S. 424

Velez Edwards, D.R. 514

Whittaker, J. 538

Wideroff, L. 495

Wolff, K. 396

Wonkam, A. 492

Worrall, B.B. 477

Zheng, X. 514

Zlot, A.I. 457 